\title{
DIGITAL WORKFLOWS FOR A 3D SEMANTIC REPRESENTATION OF AN ANCIENT MINING LANDSCAPE
}

\author{
Gerald Hiebel, Klaus Hanke \\ University of Innsbruck, Surveying and Geoinformation Unit, Innsbruck, Austria \\ (gerald.hiebel, klaus.hanke)@uibk.ac.at
}

KEY WORDS: Mining Landscape, Ontology, Semantic Technologies, Geoinformation, Information Integration

\begin{abstract}
:
The ancient mining landscape of Schwaz/Brixlegg in the Tyrol, Austria witnessed mining from prehistoric times to modern times creating a first order cultural landscape when it comes to one of the most important inventions in human history: the production of metal. In 1991 a part of this landscape was lost due to an enormous landslide that reshaped part of the mountain. With our work we want to propose a digital workflow to create a 3D semantic representation of this ancient mining landscape with its mining structures to preserve it for posterity. First, we define a conceptual model to integrate the data. It is based on the CIDOC CRM ontology and CRMgeo for geometric data. To transform our information sources to a formal representation of the classes and properties of the ontology we applied semantic web technologies and created a knowledge graph in RDF (Resource Description Framework). Through the CRMgeo extension coordinate information of mining features can be integrated into the RDF graph and thus related to the detailed digital elevation model that may be visualized together with the mining structures using Geoinformation systems or 3D visualization tools. The RDF network of the triple store can be queried using the SPARQL query language. We created a snapshot of mining, settlement and burial sites in the Bronze Age. The results of the query were loaded into a Geoinformation system and a visualization of known bronze age sites related to mining, settlement and burial activities was created.
\end{abstract}



Figure 1. Mining landscape of Schwaz in the Tyrol indicating the landslide of the Eiblschrofen in the high resolution surface model of the province of Tyrol (source: Land Tirol 2009) 


\section{INTRODUCTION}

The ancient mining landscape of Schwaz/Brixlegg in the Tyrol, Austria witnessed mining from prehistoric times to modern times (Schiebler et al. 2011). These activities left distinct physical structures in the mountains south of the Inn river ranging over $30 \mathrm{~km}$. This area documents the history of mining from bronze age copper mining to medieval and modern times silver mining creating a first order cultural landscape when it comes to one of the most important inventions in human history: the production of metal. In 1991 a part of this landscape was lost due to an enormous landslide that reshaped part of the mountain (Poisl et al. 2002) Figure 1 shows the landslide area in the high resolution surface model of the province of Tyrol (source: Land Tirol 2009).

With our work we want to propose a digital workflow to create a 3D semantic representation of this ancient mining landscape with its mining structures to preserve it for posterity. With a $3 \mathrm{D}$ semantic representation, we mean that our 2-3D georeferenced geometric information of mining landscape and structures is linked with a semantic representation of the mining structures including knowledge on functionality or periods of usage. In addition our representation includes the provenance of the information, meaning what are the sources of information and how this knowledge was obtained, if still known.

\section{DIGITAL WORKFLOWS}

\subsection{Information Sources}

The HiMAT research center of the University of Innsbruck (http://himat.uibk.ac.at) investigates the mining history of the Eastern Alps from Prehistory to Modern Times. Various projects of the research center in the area of Schwaz/Brixlegg target the location, identification and interpretation of mining structures. Geological prospections are a fundamental information source about structures originating from mining activities. Herwig Pirkl [Pirkl 1961] investigated the Schwaz/Brixlegg mining area in a detail that has not been repeated since. The result was a publication describing the geologic and surface structures of the area. It contains three geological maps in the scale 1:10000. Two of these maps have been digitized in the course of the works realized in the HiMAT research center. Structures identified by Pirkl as underground mining and surface mining have been registered together with their names and coordinates. In addition, information on mining structures provided by the Geological Survey Austria [GBA 2014] has been integrated (Figure 2).

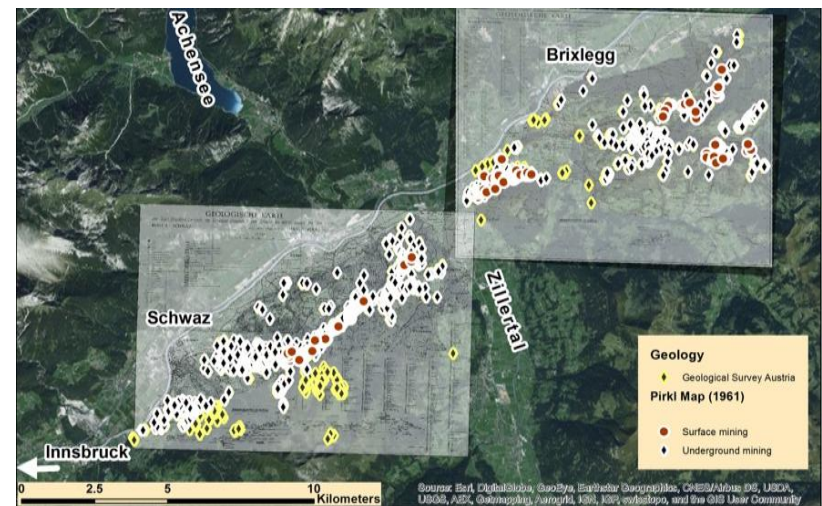

Figure 2. Mining structures identified by Pirkl and the Geological Survey Austria (source: GBA, 2014)
To understand the setting of the mining district the high resolution elevation model of the province of Tyrol is used in a $2.5 \mathrm{D}$ visualisation (Figure 3 ).

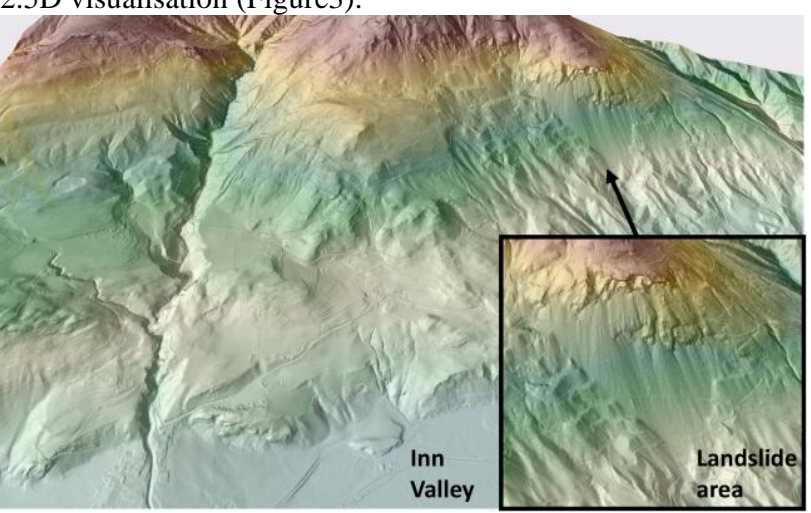

Figure 3. Landslide of the Eiblschrofen in the high resolution terrain model of the province of Tyrol (source: Land Tirol 2009)

This model is used in addition for a better location of mining structures. It was manually examined for concave and convex surface structures that are in proximity of the structures identified by Pirkl (Figure 4).

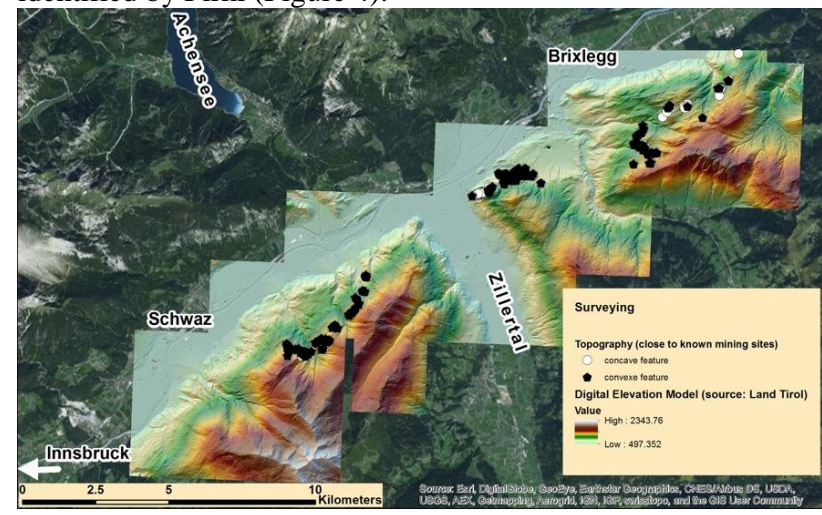

Figure 4. Surface structures identified in the high resolution elevation model of the province of Tyrol (Source: Land Tirol 2009)

HiMAT's latest project "Prehistoric copper production in the eastern and central Alps" contributed significantly to the knowledge of archaeological sites related to prehistoric mining activities through archaeological prospection and excavations. In Figure 5 these activities are illustrated together with find sites that have been extracted from archaeological literature.

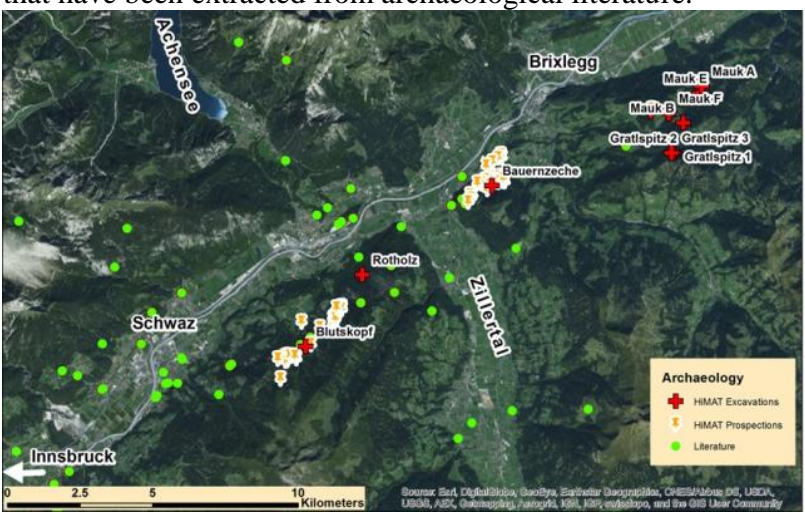

Figure 5. Archaeological sites identified in the course of HiMAT research and in archaeological literature (source: HiMAT) 
A detailed map of the area around a prehistoric mining structure called "Bauernzeche" shows the potential of integrating these sources together with a specific terrain visualization using shaded relief and $0,1 \mathrm{~m}$ contour lines. In the map we see the landscape relief and structures created through mining activities which were first observed by Pirkl and documented in geological maps and then found again by HiMAT archaeologists and documented in field survey records. We tried to detect relevant physical structures in the high resolution digital elevation model and marked areas that could correspond to the observations made by Pirkl and the HiMAT archaeologists (Figure 6).

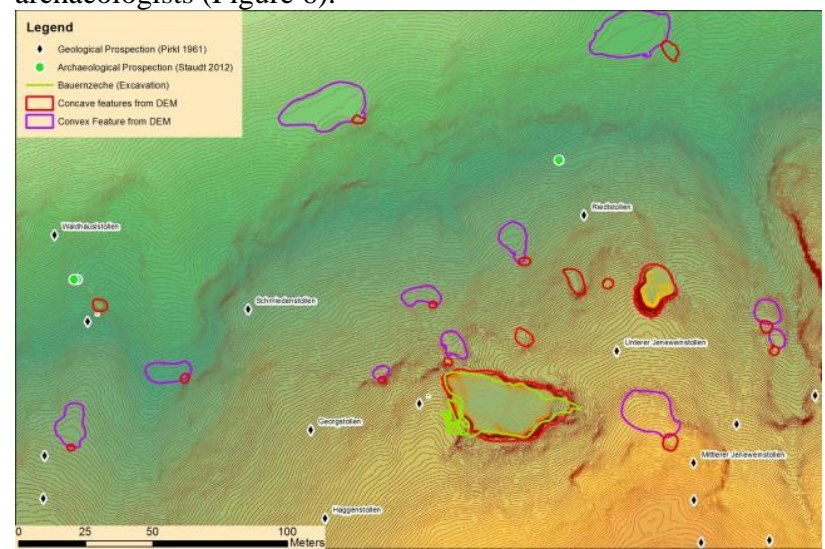

Figure 6. Information sources that indicate physical structures related to mining activities

For the data sources displayed in Figure 6 we have observations and analysis of these physical mining structures like pits, heaps, beneficiation and smelting sites. The same physical structure may have been observed or analysed several times by different humans or different scientific methodologies. In addition to the observations we have the interpretations of the physical structures as to their functionality and their dating based on observations and analysis. As shown in the illustration Geoinformation systems use layers to represent information and when used in archaeology physical structures, observations, analysis and interpretations are either mixed in one layer or there exist several layers for different information sources. On the represented area we find recent and historic observations of mining structures and there has been an excavation in the so called "Bauernzeche" where prehistoric mining activities using fire setting techniques have been verified. To integrate the information of observations, analysis and excavations together with the interpretations we need a network model that goes beyond the layer structure used in Geoinformation systems. This model should be able to represent the information available in different sources about this ancient mining landscape and build a network that can be visualised for public presentation and explored for further research.

\subsection{Conceptual model to link semantics with geoinformation}

The challenge is to integrate these different information sources coming from surveying, archaeology, geology and history under one conceptual model and find a digital workflow resulting in a formal representation that is able to hold the relevant information of the different sources together with the geometric information. Information integration with spatial data using GIS and relational databases has been successfully pursued in the HiMAT research center (Hiebel et al. 2013) and the presented approach is the continuation of these efforts. One goal is the localization, identification and interpretation of mining structures in the targeted landscape that can be represented and visualized within a detailed $2.5 \mathrm{D}$ digital elevation model. A semantic database provides access to information available on specific mining structures and links to 3D models and visualizations of selected structures. The digital workflow starts with defining a conceptual model to integrate the data.

We choose the CIDOC CRM ontology [Le Boeuf et al. 2016] because it is an ISO standard in Cultural Heritage Documentation and an event centric data model. Ancient mining activities as well as the mining structures and landscape they produced are modelled using classes of the CIDOC CRM.

Extensions of the CIDOC CRM (CIDOC CRM 2016) were used to model contemporary research activities (which are subclasses of events) creating information sources and geometric information. Observations and Interpretations were modelled using CRMsci, the creation of geometric information was modelled using CRMdig and the geometric information itself was modelled with CRMgeo (Hiebel et al. 2016). Examples for using CIDOC CRM extensions to model information sources in mining history and integrate them on an interdisciplinary level have been presented in Hiebel et al. 2014. Figure 7 illustrates the conceptual model for the current approach.

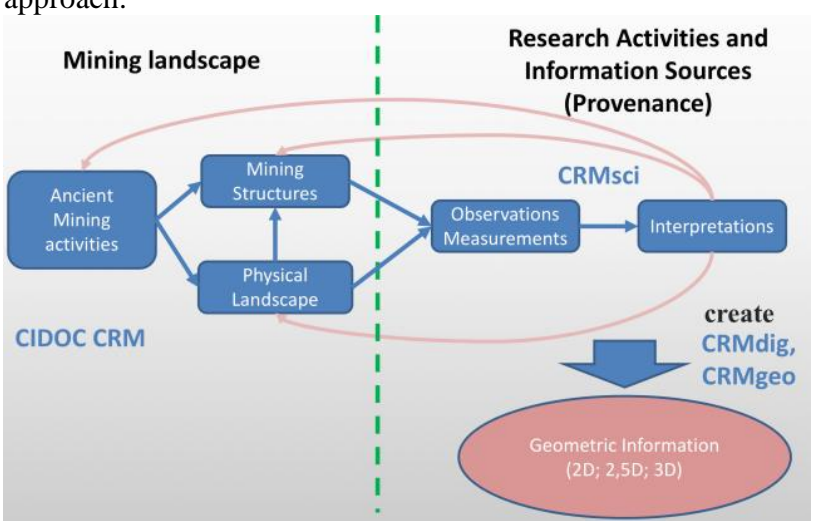

Figure 7. Relation of Mining Landscape, Information Sources and Geometric Information in a network model

The classes of the model had to be refined with a thesaurus (Figure 8) in order to represent the detailed information of the available documentation, to answer relevant research questions and to create appropriate visualisations.

\section{Research Activities and Information Sources}

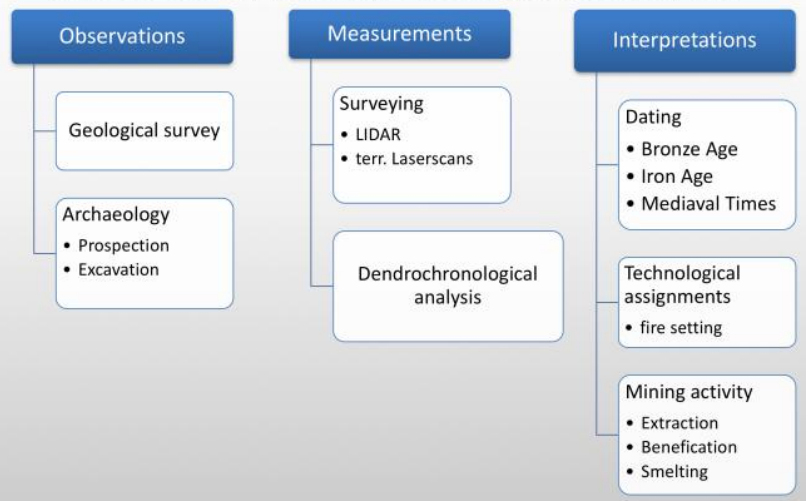

Figure 8. Refinement of Research Activities and Information Sources in a thesaurus

The integration of vocabularies originating from different sources is a serious challenge (Doerr 2006). Within the DARIAH Infrastructure (www.dariah.eu) an approach was 
developed to integrate terms within a backbone thesaurus and thus create the ability to query upper levels without the need to reach consensus on lower level terms which is often an almost impossible task to accomplish (Dariah EU 2016).

In Figure 9 we want to show how to apply our model on the past human activity of ore extraction through fire setting. This mining activity with the specific technology created the physical structure of mine "Mauk E". It was excavated and dendrochronological measurements have been conducted on wood samples, as well as a 3D documentation of the mine and the surrounding terrain took place. The interpretation from observations and measurements state that ore extraction took place between 720 to 707 B.C. and that the fire setting technology was used.

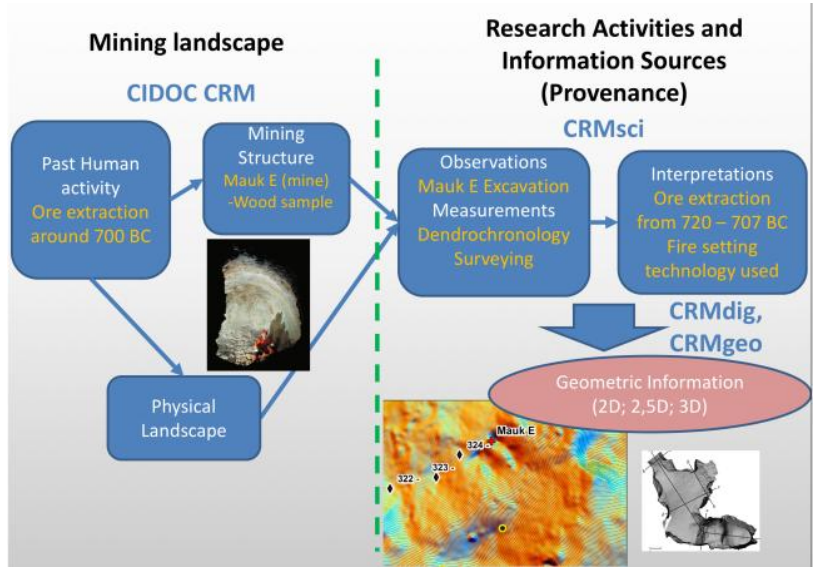

Figure 9. Example of the modeling for the mine "Mauk E"

\subsection{Implementation}

To transform our information sources to a formal representation of the classes and properties of the ontology we applied semantic web technologies and created a knowledge graph in RDF (Resource Description Framework), a data format that is able to relate logical statements within a network (W3C 2014). $\mathrm{RDF}$ is the foundation of the Linked Open Data (LOD) Cloud, where data sets are linked to each other on a global level. Using this format and technology means that the information within the semantic database can be linked to Linked Open Data resources like Wikipedia or Geonames. Through the CRMgeo extension coordinate information of mining features can be integrated into the RDF graph and thus related to the detailed digital elevation model that may be visualized together with the mining structures using Geoinformation systems or 3D visualization tools. The available information has to be converted in a structured format, either being a tabular format, a relational database or an XML structure. The terms used in the existing documentation have to be aligned to the thesaurus to obtain data that can be mapped to the classes of our ontology using the specialisations provided by the thesaurus. We used Karma [ISI 2016], a tool of the semantic web community to map the information sources, enriched with thesaurus terms to the formal definitions of the CIDOC CRM ontology. A knowledge graph is created to represent the information which can be exported in RDF. The thesaurus was created with the Karma tool as well and represented in SKOS (Simple Knowledge Organization System), a data model of the semantic web community for sharing and linking knowledge organization systems, such as thesauri, taxonomies, classification schemes and subject heading systems (W3C 2009).
After mapping the different information sources and the thesaurus to the common data model the created RDF structure is ingested in a triple store, which is a database to store RDF data. In the triple store the linking of the resources (single information source elements like a specific underground mine or a concept like the Early Bronze Age) takes place performing the actual integration. Resources are either linked on a class level (because they belong to the same CIDOC CRM class, e.g. Observation), on the SKOS concept level (because the same thesaurus term was attributed to them, e.g. "Bronze Age") or on an individual level (because they describe the same material structure object or observation, e.g. "Barbarastollen"). Linking on an individual level is also known as co reference or entity matching and may involve additional processes to assess the identity of individuals if no common identifier is available in the different data sources, which is often not the case.

\section{DIGITAL MINING LANDSCAPE}

The RDF network of the triple store can be queried using the SPARQL query language. We created a snapshot of mining, settlement and burial sites in the Bronze Age, differentiating between ore extraction, ore processing and smelting activities to test the semantics of our representation. The results of the query were loaded into a Geoinformation system and a 3D visualization of known bronze age sites related to mining, settlement and burial activities was created.

The integration of the information and the subsequent provision in adequate tools can help researchers of prehistoric mining archaeology significantly to answer their research questions and document the features of our mining landscape for posterity.

The RDF network of the triple store can be queried using the SPARQL (W3C 2013) query language. To show the potential of the integration we used one of our research questions. concerning the location of mining, settlement and burial sites in the Bronze Age. In addition we wanted to differentiate between sites of ore extraction, ore processing and smelting activities. As we wanted to show as well potential sites of Bronze Age ore extraction we display in addition sites were the fire setting technology was used but no additional dating analysis has been carried out at the sites. The results of the query were loaded into a Geoinformation system and a map of known and potential bronze age sites related to mining, settlement and burial activities was created (Figure 10).

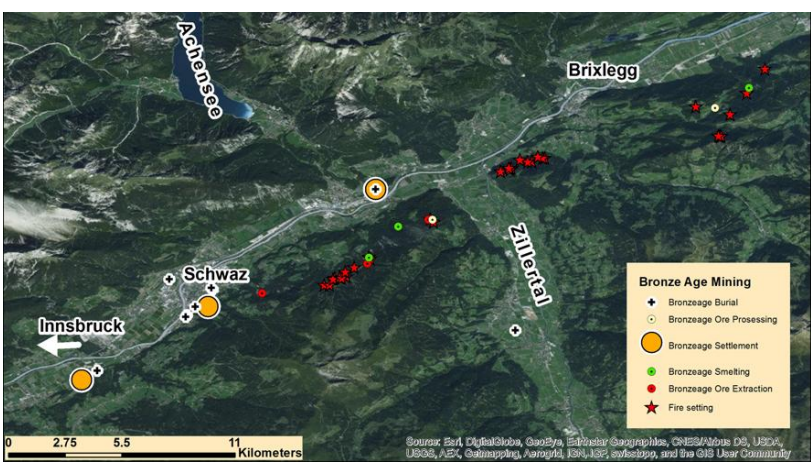

Figure 10. Map of known bronze age sites related to mining, settlement and burial activities together with sites where fire setting technology was used

\section{CONCLUSION AND OUTLOOK}

We developed an approach to integrate information related to the field of prehistoric mining archaeology coming from various 
sources by developing a common data model and using tools and specifications of the semantic web community to perform the actual integration. With a sample query we could show that the integration process works and that the triple store can be used to answer specific research questions.

Within the activities of the CIPA Task Group "Ontology" (http://cipa.icomos.org/portfolio-item/task_group_ontology/) a tutorial will be made that documents the digital workflow to create such a 3D Semantic Representation. The tutorial will contain exemplary data from the mining landscape and mining structures and the detailed steps to transform the data to RDF using a CIDOC CRM structure. Finally RDF data are loaded into a triple store and sample queries show how results can be obtained that may be loaded into a Geoinformation system. All tools used in the process are open source.

With this use case on an ancient mining landscape and the tutorial we want to promote the use of ontologies and semantic technologies and show how they can be integrated with 2-3D geometric data and visualizations.

\section{ACKNOWLEDGEMENTS}

The research presented here was financed by the Austrian Science Fund in the course of an Erwin Schrödinger scholarship (J 3646-N15) and by the University of Innsbruck in the course of a graduate scholarship (240346).

\section{REFERENCES}

CIDOC CRM, 2016. CIDOC CRM Compatible models \& Collaborations, http://www.cidoc-crm.org/collaborations (9.1.2017)

Dariah EU, 2016. DARIAH Backbone Thesaurus (BBT) Definition of a model for sustainable interoperable thesauri maintenance, Produced by the Thesaurus Maintenance Working Group, VCC3, DARIAH EU, http://83.212.168.219/DariahCrete/sites/default/files/dariah_bbt _v_1.2_draft_v4.pdf (4.1.2017)

Doerr, M., 2006. Semantic Problems of Thesaurus Mapping. Journal Of Digital Information, 1(8). Retrieved from https://journals.tdl.org/jodi/index.php/jodi/article/view/31/32 $(3.11 .2016)$

GBA, 2014. Digitale Datensätze des Bergbau/Haldenkatasters betreffend ausgewählter Bergbaugebiete im Raum SchwazBrixlegg und Kitzbühel-Jochberg, Fachabteilung Rohstoffgeologie der Geologischen Bundesanstalt

Hiebel, G., Hanke, K. and Hayek, I., 2013. Methodology for CIDOC CRM Based Data Integration with Spatial Data. In: F. Contreras, M. Farjas, and F. J. Melero. 2013. CAA 2010: Fusion of Cultures. Proceedings of the 38th Annual Conference on Computer Applications and Quantitative Methods in Archaeology, Granada, Spain, April 2010 - BAR International. British Archaeological Reports, UK. ISBN: 978-84-693-0772-4 P. 547-554.

Hiebel, G., Doerr, M., Eide Ø., 2016. CRMgeo: A Spatiotemporal Extension of CIDOC-CRM in International Journal on Digital Libraries doi:10.1007/s00799-016-0192-4

Hiebel, G., Doerr, M., Hanke K., Masur, A., 2014. How to put archaeological geometric data into context? Representing mining history research with CIDOC CRM and extensions in: Ioannides, M., (eds.). International Journal of Heritage in the
Digital Era: Special Issue on Metadata, Semantics and Ontologies for Cultural Heritage, Cyprus University of Technology, Limassol, Cyprus

ISI, 2016. Karma: A Data Integration Tool, http://www.isi.edu/integration/karma/ (3.11.2016)

Le Boeuf, P. Doerr, M. Ore, CH.E. Stead. S., 2016. Definition of the CIDOC Conceptual Reference Model, http://www.cidoccrm.org/official_release_cidoc.html (6.4.2016).

Pirkl, H., 1961. Geologie des Trias-Streifens und des Schwazer Dolomits südlich des Inn zwischen Schwaz und Wörgl (Tirol), Jahrbuch Geol. B. A. (1961), Bd. 104. 1. Heft, (Wien 1961)

Poisel R.; Roth W.; Preh A.; Tentschert E.; Angerer H., 2002: The Eiblschrofen rock falls; interpretation of monitoring results of a complex rock structure. in J. Rybar, J. Stemberk, P. WagnerLandslides: Proceedings of the First European Conference on Landslides, Prague, Czech Republic, 24-26 June 2002, Pages 449-454 2002, ISBN 9789058093936

Schibler, J., Breitenlechner, E., Deschler-Erb, S., Goldenberg, G., Hanke, K., Hiebel, G., . . . Oeggl, K., (2011). Miners and mining in the Late Bronze Age: A multidisciplinary study from Austria. Antiquity,85(330), $1259-1278$ doi:10.1017/S0003598X00062049 (14.06.2017)

W3C, 2009. SKOS Simple Knowledge Organization System Reference. https://www.w3.org/TR/2009/REC-skos-reference20090818/ (19.6.2016)

W3C, 2013. SPARQL $1.1 \quad$ Overview https://www.w3.org/TR/sparql11-overview/ (9.1.2017)

W3C, 2014. Resource Description Framework (RDF) http://www.w3.org/RDF/ (19.6.2016) 